\title{
CFD 2D Description of Local Flow of Polymer Workpiece through a modified U-Shaped Die During Equal Channel Multiple Angular Extrusion
}

\author{
Alexander V. Perig ${ }^{a *}$, Nikolai N. Golodenko ${ }^{b}$ \\ ${ }^{a}$ Manufacturing Processes and Automation Engineering Department, Donbass State Engineering \\ Academy, Shkadinova Str., 72, 84313, Kramatorsk, Ukraine \\ ${ }^{b}$ Department of Water Supply, Water Disposal and Water Resources Protection, Donbass National \\ Academy of Civil Engineering and Architecture, Lazo Str., 14, 84333, Kramatorsk, Ukraine
}

Received: January 8, 2016; Revised: February 18, 2016; Accepted: March 11, 2016

\begin{abstract}
The present article is focused on a 2D computational fluid mechanics study of local viscous flow dynamics and the formation character of rotary modes of deformation during Equal Channel Multiple Angular Extrusion (ECMAE) of a polymer workpiece fluid model through a U-shaped die with parallel slants in channel intersection zones. The present local flow problem was experimentally analyzed using physical simulation methods and theoretically studied with numerical fluid mechanics techniques. The computational approach has been grounded on the numerical finite difference solution of the boundary value problem for the Navier-Stokes equations in the curl transfer form for the local viscous flow of incompressible Newtonian fluid through a U-shaped rectangular die with parallel slants. The derived research results allow us to draw a conclusion that the implementation of a geometric design of parallel slants within a 2-turn U-shaped die results in localization of the maximum tangential stresses within the workpiece volume to the vicinity of these parallel slants during ECMAE.
\end{abstract}

Keywords: Equal Channel Multiple Angular Extrusion, Macroscopic Rotation, U-shaped Die, Physical Simulation, Initial Circular Grids, Navier-Stokes equations, Curl Transfer Equation, Boundary-Value Problem, Finite Difference Solution

\section{Introduction}

Computational Fluid Dynamics (CFD) techniques ${ }^{(3-11)}$ are finding new and important industrial applications in the materials science related fields of Severe Plastic Deformation (SPD) ${ }^{(1,3,5-10)}$. Minakowski (2014) has applied CFD-based techniques to a mathematical description of the flow of an aluminum workpiece during Equal Channel Angular Extrusion (ECAE) through a 2-turn angular die with internal and external radii in the channel intersection zones ${ }^{(3)}$. Perig et al., have solved the Navier-Stokes equations in a curl transfer form for the ECAE problem of workpiece viscous flow ${ }^{(5-8)}$. Perig and Golodenko have applied CFD techniques to the numerical solution of the $2 \mathrm{D}$ problem for viscous material ECAE through a 2-turn multiple-angle S-shaped die with a movable inlet wall ${ }^{(6)}$. Perig et al., have applied a CFD-based method of numerical integration of a curl transfer equation for viscous flow of a workpiece through a die with parallel slants in the channel intersection zone ${ }^{(7)}$. Rejaeian and Aghaie-Khafri (2014) have applied a CFD-based stream function technique to a velocity field derivation in the channel intersection zone during ECAE of a workpiece through an angular die with external and internal radii in the channel intersection zone ${ }^{(10)}$. At the present time further development of SPD processing schemes during Equal Channel Multiple Angular Extrusion (ECMAE) is grounded on further increases in workpiece length and increases in accumulated shear deformations within the worked material

*e-mail: olexander.perig@gmail.com, nik_nik_Gold@mail.ru volume by increasing the quantity of deformation zones for every pass of ECMAE ${ }^{(1,6,7)}$.

The principal goal of ECMAE pressure working of polymer materials is focused on the introduction and accumulation of large plastic deformations in the polymer workpiece for further fragmentation of lamellar polymer structure with mechanical properties enhancement while retaining the optical transparency of processed polymer workpiece $^{(1,4-8)}$. Possible applications for ECMAE worked polymers are associated with aeronautical and chemical industry equipment production ${ }^{(1,4-8)}$.

The solution of the above mentioned technological problems is within the aims and scopes of research, conducted by Beloshenko et al., (2009) ${ }^{1}$, Minakowski (2014) ${ }^{3}$ Perig et al. $\left.{ }^{(2014)}\right)^{(5-9)}$, and Rejaeian and Aghaiae-Khafri (2014) ${ }^{(10)}$, and a number of other researchers ${ }^{(2,4,12,13)}$.

The 2-turn, 3-turn, ..., and n-turn ECMAE dies with geometries of $\mathrm{S}-, \mathrm{U}-, \mathrm{T}-, \mathrm{X}-, \mathrm{W}-$ types could be classified as multiple angle dies ${ }^{(1,3,6,7)}$. So the character of local flow of workpieces during ECMAE is determined both by geometric features of the die tooling and by the rheology of deformable metal ${ }^{(10)}$, polymer ${ }^{(1,3-8)}$, powder, and composite materials. Both the die geometry (Figs. $(1-2))$ and the workpiece rheology (Figs. ${ }^{(3,4)}$ ) determine the kinematic and dynamic features of the local flow, rotation angles of the principal axes of the flow, and the heterogeneity of material rotation during workpiece forming through the U-die. 

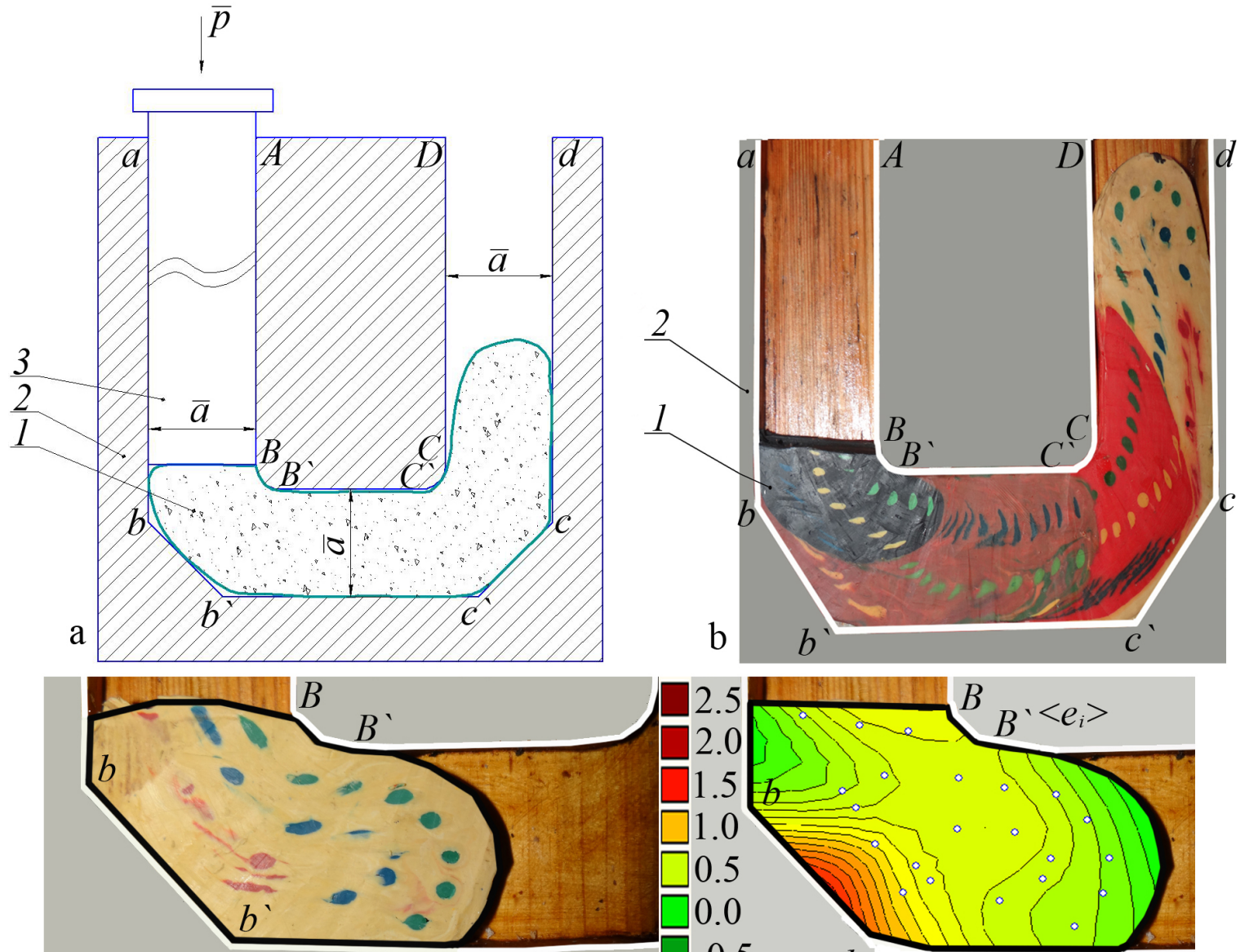

$\mathrm{c}$

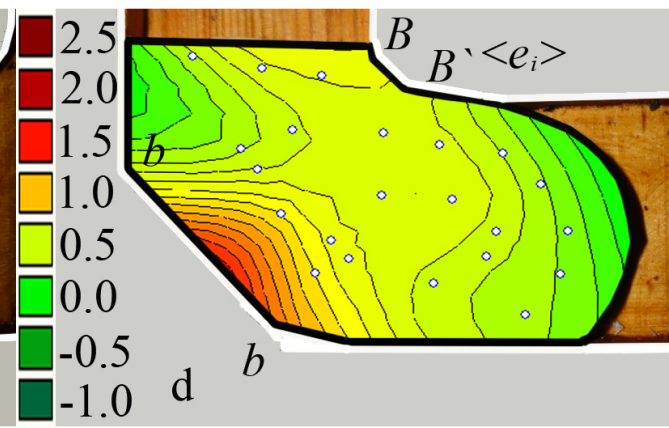

Fig. 1. ECMAE schemes of a truncated U-shaped die (1 - workpiece; 2 - split die; 3 - punch): (a) - scheme of the flow of the workpiece; (b) - workpiece physical model with the initial circular gridlines at the final stage of filling the outlet channel cdCD of the U - shaped die; (c) - the soft physical model of deformable workpiece with the initial circular gridlines at the initial stage of filling the transition channel bb'BB' of the U - shaped die; (d) - the experimentally-theoretical field of logarithmic strain intensities for the one-component model (c).

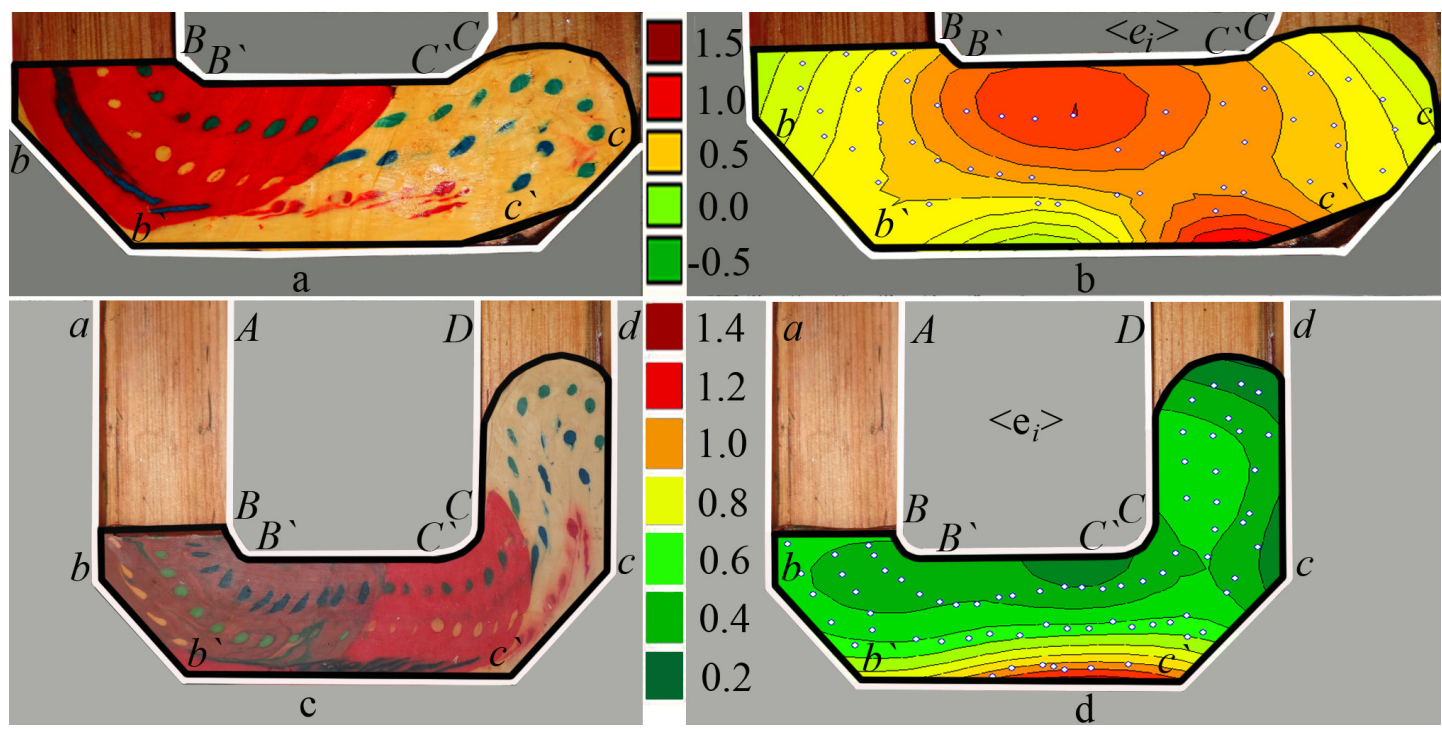

Fig. 2. ECMAE schemes of a truncated U-shaped die: (a, c) - soft workpiece models; (b, d) - the experimentally-theoretical fields of logarithmic strain intensities for the two- (a) and three- (c) component models (a, c). 
However the dynamics of macroscopic rotation during workpiece ECMAE through a U-shaped multiple-angle die is still not fully addressed in the previous known research (1,3-10), which emphasizes the importance and novelty of the present article.

The objective of the present article is the estimation of kinematic and dynamic features of workpiece flow in the channel intersection zones of a truncated U-shaped multiple-angle die with parallel slants, accounting for hydrodynamic effects of workpiece material viscosity.

\section{Aims and Scopes of the Article. Prime Novelty Statement of Research}

The present article is focused on the experimental and theoretical description of viscous workpiece flow through a U-shaped die with parallel slants in channel intersection zones.

The aim of the present research is the phenomenological continuum mechanics based description of viscous workpiece flow through a U-shaped die with parallel slants in channel intersection zones.

The subject of the present research is the process of ECAE working through a U-shaped die with parallel slants in channel intersection zones with viscous flow of polymeric workpiece models, forced by the external action of standard rectangular punch shapes.

The object of the present research is to establish the characteristics of the viscous flow of workpiece models through the U-shaped die with parallel slants in channel intersection zones with respect to workpiece material rheology in the viscous ECAE process.

The experimental novelty of the present article is based on the introduction of initial circular gridlines to study the influence of slanted U-shaped die on viscous workpiece ECMAE flow.

The prime novelty of the present research is the numerical finite-difference solution of boundary value problem for Navier-Stokes equations in the curl transfer form for the viscous workpiece flow through the U-shaped die with parallel slants in channel intersection zones.

\section{Physical Simulation Study of Viscous Flow through a U-shaped die with parallel slants in channel intersection zones}

To solve the posed problem experimentally we will analyze the workpiece local flow features during ECMAE through an introduction of such physical simulation techniques as the marker method and the method of initial circular gridlines (Figs. $(1-2))$.

Plasticine based physical simulation experiments in Figs. $(1-2)$ are the new original experimental results, derived personally by the authors.

The experimental values of the Coulomb friction coefficient and the plastic friction factor in the Siebel (Tresca) friction law have been estimated with plasticine rings upsetting between two wooden plates. The plasticine rings were made with the following ratio of dimensions $D: d: H=6: 3: 2$, where $D$ is external diameter of plasticine ring, $d$ is internal diameter of plasticine ring, and $H$ is the height of plasticine ring ${ }^{(12,13)}$. It was experimentally determined by authors that the Coulomb friction coefficient is $f \approx 0.181\left(\tau_{f}=f^{\bullet} \sigma_{n}\right)$ and the plastic friction factor in the Siebel (Tresca) friction law is $m \approx 0.594\left(\tau_{f}=m \bullet k ; k=\sigma_{S} / 3^{0.5}\right)$ for contact pair "wooden die model-plasticine workpiece model", where $\tau_{f}$ is the friction stress; $\sigma_{n}$ is the normal stress; $k$ is the maximum tangential stress for plasticine material with flow stress $\sigma_{S}$, i.e. the plastic constant $k$ is the shear strength of the extruded material.

The initial plasticine physical models of the workpieces in Figs. $(1-2)$ were made in the shapes of rectangular parallelepipeds. These rectangular parallelepipeds were frozen, the front sides of parallelepipeds were marked, and through-holes in parallelepipeds were perforated. Then the plasticine parallelepipeds with through-holes were frozen again. Simultaneously, plasticine pieces of different colors were heated to the half-solid state and placed into the through-holes of parallelepipeds through the application of squirt without needle. In this way the initial workpiece model was made in the shape of a rectangular parallelepiped and the initial circular plasticine gridline was formed within the plasticine model of the workpiece (Figs. $(1-2)$ ).

Simple new original physical simulation experiments of workpiece model flow during ECMAE through a truncated U-shaped die with the channel width $\bar{a}=40 \mathrm{~mm}$ are shown in Figs. $(1-2)$. The ECMAE truncated U-shaped die model is fabricated from wood in Figs. $(1-2)$, with the front part of the ECMAE die model fabricated with glassy plexiglass. In Figs. $(1-2)$ are outlined the plasticine physical models with circular plasticine gridlines.

In Fig. 1 are shown ECMAE schemes (Fig. 1(a, b, c)) for pressing soft physical models of workpieces through a truncated U-shaped die (1 - workpiece; 2 - split die; 3 - punch), where channel width is $\bar{a}=40 \mathrm{~mm}$; the current values of workpiece lengths are $\bar{L}_{I} \approx 2 \cdot \bar{a}=80 \mathrm{~mm}$ in Fig. 1(c, d) and $\bar{L}_{I V} \approx 6.7 \cdot \bar{a}=268 \mathrm{~mm}$ in Fig. 1(b), where suffix numbers I and IV denote that the current physical model of the workpiece consists of one- Fig. 1(c, d) and four- Fig. 1(b) components (Fig. 1).

In Fig. 2 are shown physical simulation-derived schemes of the local flow of the soft physical models of deformable workpiece with the initial circular gridlines at the final stage of filling the transition channel $b^{\prime} c^{\prime} B^{\prime} C^{\prime}$ ' (Fig. 2(a, b)) and at the initial stage of filling the outlet channel $c d C D$ (Fig. 2(c, d)) of the $U$ - shaped die, where channel width is $\bar{a}=40 \mathrm{~mm}$; the current values of workpiece lengths are $\bar{L}_{I I} \approx 3.5 \cdot \bar{a}=140 \mathrm{~mm}$ (Fig. 2(a, b)) and $\bar{L}_{I I I} \approx 5.3 \cdot \bar{a}=212 \mathrm{~mm}$ (Fig. 2(c, d)), where suffix numbers II and III denote that the current physical model of the workpiece consists of two- (Fig. 2(a, b)) and three- (Fig. 2(c, d)) components.

The physical model of the long workpiece consisted of a 4-part model, which was formed by successive extrusion of the 4 initial short parts through the truncated U-shaped die $a b b^{\prime} c^{\prime} c d-A B B^{\prime} C^{\prime} C D$ (Figs. (1(b, c) \& 2(a, c))).

As the total workpiece length increases, the viscous workpiece material successively fills the inlet channel $a b A B$ (Fig. 1a), the transition channel $b^{\prime} c^{\prime} B^{\prime} C^{\prime}$ (Figs. (1(c, d) \& 2(a, b))), and the outlet channel $c d C D$ (Fig. 2(c,d)) of the U-shaped multiple-angle die. 
The corresponding diagrams of logarithmic strain intensities $\left\langle e_{i}\right\rangle$ have been derived on the basis of the experimental results (Figs. (1(b, c) \& 2(a, c))) and are shown in Fig. 1d and Fig. 2(b, d). Derived diagrams of $\left\langle e_{i}\right\rangle$ show the character of successive displacement of the zones with the localization of maximum values of $\left\langle e_{i}\right\rangle$ during workpiece ECMAE through a U-shaped die from the transition truncated die zone $b b^{\prime} B B^{\prime}$ (Figs. $(1-2))$ to the zone of the transitional die channel $b^{\prime} c^{\prime} B$ ' $C$ ' (Figs. $\left.(1-2)\right)$. The averaged values of experimental strain intensities $\left\langle e_{i}\right\rangle$ are: 0.490 in Fig. $1 d$; 0.458 in Fig. 2b, and 0.839 in Fig. 2d. This shows increasing values of $\left\langle e_{i}\right\rangle$ as further deformation of the workpiece occurs through the truncated U-shaped die in Figs. $(1-2)$.

We will introduce the term "average unevenness of logarithmic strain" as

$\left\langle e_{i S U}\right\rangle=\frac{1}{2}\left(\frac{\left(e_{i \max }-\left\langle e_{i}\right\rangle\right)}{e_{i \max }}+\frac{\left(e_{i \max }-\left\langle e_{i}\right\rangle\right)}{\left\langle e_{i}\right\rangle}\right)$

The averaged values of experimental unevenness of logarithmic strain $\left\langle e_{i S U}\right\rangle$ are: 1.07 in Fig. 1d; 0.892 in Fig. 2b, and 12.341 in Fig. $2 \mathrm{~d}$. This shows increasing values of $\left\langle e_{i S U}\right\rangle$ as further deformation of the workpiece occurs through the truncated U-shaped die to the outlet die channel $c d C D$ in Fig. 2c. The complex nature of the local flow of the workpiece material through the U-shaped die is additionally characterized by such deformation parameters as the degree of "mixing", macroscopic rotation, and rotation non-uniformity. We will define the degree of workpiece material "mixing" in Figs. (1(b, c) \& 2(a, c)) as the inclination angle $\alpha$ of the major axis of the deformable elliptical marker with the directions $a b, b b^{\prime}, b^{\prime} c^{\prime}, c^{\prime} c, c d$ of the workpiece flow. We will define the macroscopic rotation in Figs. $(1(b, c) \& 2(a, c))$ as the inclination angle increment $\Delta \alpha$ of the marker major axis with the directions $a b, b b^{\prime}, b^{\prime} c^{\prime}, c^{\prime} c, c d$ of the workpiece flow. Experimentally measured average values of the degree of workpiece material "mixing" with and without accounting of algebraic signs of $\alpha$ have the following values: $\langle\alpha\rangle=20.541^{\circ}$ and $\left\langle|\alpha|>=20.246^{\circ}\right.$ in Fig. $1 \mathrm{~d} ;\langle\alpha\rangle=\left\langle|\alpha|>=42.824^{\circ}\right.$ in Fig. $2 \mathrm{~b}$, and $\langle\alpha\rangle=36.887^{\circ}$ and $\left\langle|\alpha|>=36.685^{\circ}\right.$ in Fig. 2 d. These derived experimental results show the general increase in workpiece material "mixing" as further deformation of the workpiece occurs through the truncated U-shaped ECMAE die to the outlet die channel $c d C D$.

We will define rotation non-uniformity of the material as the formation of inflection points and "intakes" within the elliptical markers. Such experimental patterns are visually observable at sections of the workpiece physical models in Figs. (1(b, c) \& 2(a, c)).

\section{CFD Simulation of Viscous Flow through a U-shaped die with parallel slants in channel intersection zones}

We will apply a CFD-based technique Figs. $(3-4)$ for a quantitative phenomenological description of macroscopic rotation dynamics, experimentally observable in Figs. (1(b, c) \& 2(a, c)). We will introduce the Navier-Stokes equations for the CFD description of viscous flow of the worked material physical models through the U-shaped slanted die $a b b^{\prime} c^{\prime} c d-A B B^{\prime} C^{\prime} C D$ in Figs. $(1(\mathrm{~b}, \mathrm{c}) \& 2(\mathrm{a}, \mathrm{c}))$.
The theoretical background for the use of the Navier-Stokes equations in the present article (Figs. $(3-4)$ ) is as follows. For the anomalous or non-Newtonian liquids like plasticine models of polymer workpieces in Figs. $(1(b, c) \& 2(a, c))$, the law of internal friction is defined by the Bingham equation as $\tau=\tau_{0}+\eta_{v i s} \cdot(d w / d z)$, where $\tau_{0}$ are the tangential stresses within the fluid at rest ${ }^{4}$. The "plasticine" liquid in Figs. $(1-2)$ can flow only after overcoming the $\tau_{0}$ tangential stresses. Then for $\tau>\tau_{0}$ we have that $\tau_{\mathrm{s}}=\eta_{v i s} \cdot(d w / d z)$, where $\tau_{\mathrm{S}}=\tau-\tau_{0}$ is the degree to which the tangential stresses exceed the fluidity level. In this work we deal with the steady flow regime through the die $a b b^{\prime} c^{\prime} c d-A B B^{\prime} C^{\prime} C D$, when the fluidity level is overcome (Figs. $(3-4))$. So for the Bingham fluid we have the same Navier-Stokes equations as in the case of a Newtonian liquid, but the computational values of the tangential stresses in reality are the overcoming of the tangential stresses over the fluidity level. Taking into account all these considerations in this work we are using the Navier-Stokes equations as the first ideal viscous approach to polymer flow during ECMAE through the U-shaped slanted die $a b b^{\prime} c^{\prime} c d-A B B^{\prime} C^{\prime} C D$ in Figs. $(1(\mathrm{~b}, \mathrm{c}) \& 2(\mathrm{a}, \mathrm{c}))$. In order to derive the mathematical model of the viscous material flow during ECMAE through the U-shaped slanted die $a b b^{\prime} c^{\prime} c d-A B B^{\prime} C^{\prime} C D$ in Figs. $(1-2)$ we will apply the Navier-Stokes equations [4-8, 11]. To use both dimensional and dimensionless values of physical variables, we will mark dimensional values with overline symbols.

The curl transfer equation in dimensionless variables will have the following form [11]:

$$
\frac{\partial \zeta}{\partial t}=-\operatorname{Re}\left(\frac{\partial(u \zeta)}{\partial x}+\frac{\partial(v \zeta)}{\partial y}\right)+\left(\frac{\partial^{2} \zeta}{\partial x^{2}}+\frac{\partial^{2} \zeta}{\partial y^{2}}\right)
$$

where the dimensionless curl function $\zeta$ (Fig. 3b) will be defined as:

$\zeta=\frac{\partial u}{\partial y}-\frac{\partial v}{\partial x}$

Equations (2)-(3) we write in finite-difference form for further numerical integration according to the method of alternating directions. Flow function $\psi$ (Fig. 3c) we find with Richardson iteration method. This article deals with a numerical estimate of the U-shaped slanted die geometric effect on viscous polymer planar flow, based on a mathematical simulation of a Newtonian incompressible fluid motion through a modified $a b b^{\prime} c^{\prime} c d-A B B^{\prime} C^{\prime} C D$ die with truncated deformation zones $b b$ ' and $c^{\prime} c$.

The numerical solution of the viscous flow problem for material flow in a U-shaped ECMAE slanted die (Figs. (1 - 2)) requires the formulation of a boundary value problem for the Navier-Stokes equation in the curl transfer form (2)-(3) for $\partial \zeta / \partial t=-\operatorname{Re}(\partial(u \zeta) / \partial x+\partial(v \zeta) / \partial y)+\partial^{2} \zeta / \partial \mathrm{x}^{2}+\partial^{2} \zeta / \partial \mathrm{y}^{2}$, where $\zeta=\partial \mathrm{u} / \partial \mathrm{y}-\partial \mathrm{v} / \partial \mathrm{x}$ (Fig. 3b). We now study the steady-state regime of viscous flow for a physical model of polymer material (Figs. $(3-4))$. So the initial conditions we will assume in the form of a rough approximation of the stationary solution (Figs. $(3-4)$ ). For steady flow of a viscous material through a modified ECMAE die $a b b^{\prime} c^{\prime} c d-A B B^{\prime} C^{\prime} C D$, the initial conditions are as follows:

$\zeta_{i, j}^{0}=1 ; u_{i<60, j<20}^{0}=1 ; u_{i<60, j>60}^{0}=-1$ 

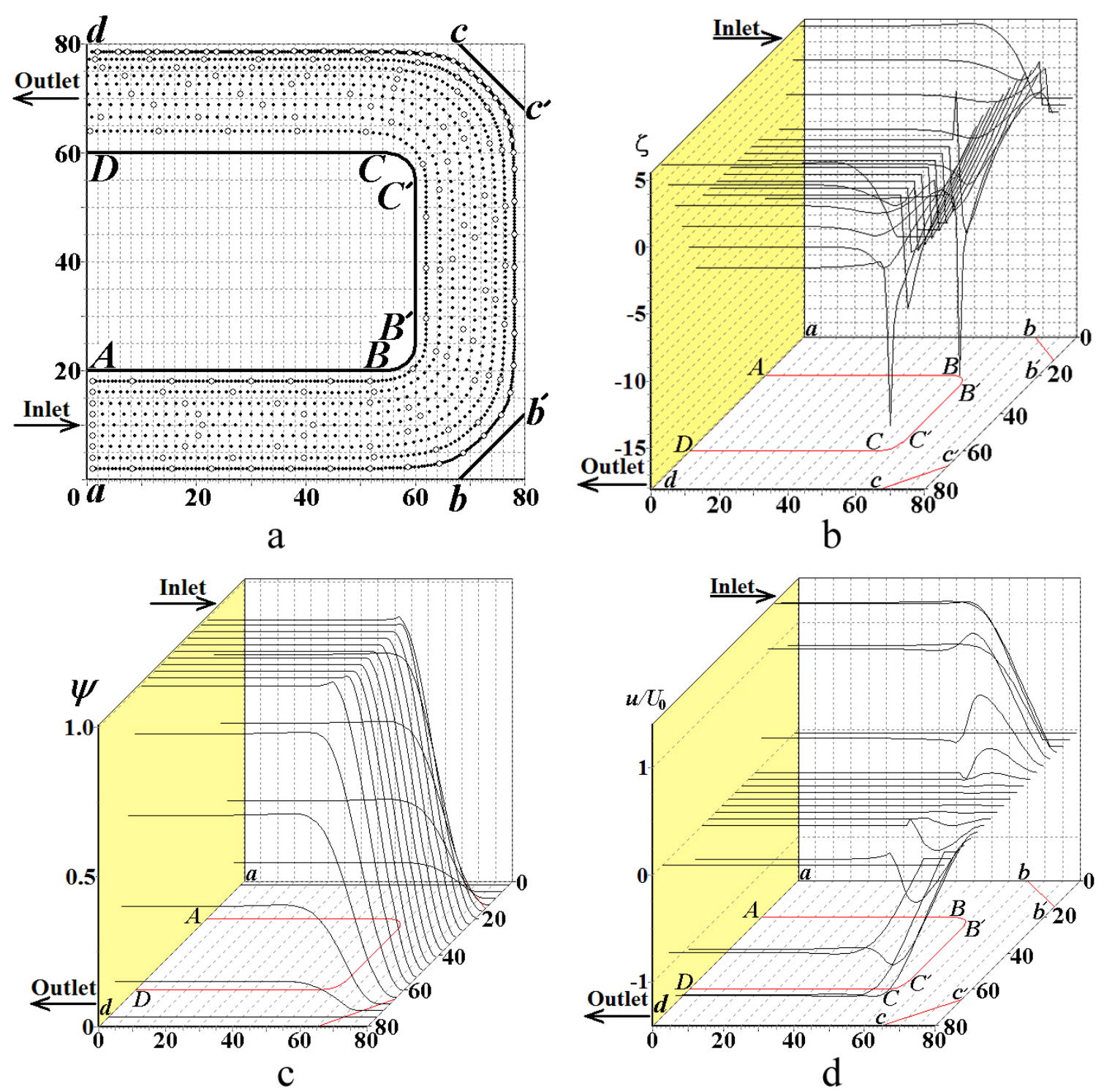

Fig. 3. CFD-derived flow lines (a), curl function $\zeta$ (b), flow function $\psi$ (c), and dimensionless u-component of flow velocity w (d), derived for ECMAE of plasticine viscous fluid through the U-shaped slanted die abb'c'cd - ABB'C'CD, where the entrance $(\mathrm{aA})$ and the exit $(\mathrm{dD})$ are at the left.

Die channel wall boundary conditions (BCs) are defined as the sticking of viscous material to the surface of die $a b b^{\prime} c^{\prime} c d-A B B^{\prime} C^{\prime} C D$ walls. So for the die walls the BCs are as follows:

for the nodes at the stream boundary $\left(A B B^{\prime} C^{\prime} C D\right)$ we have

$\psi_{i, j}=1 ; \zeta_{i, j}=0 ; u_{i, j}=0 ; v_{i, j}=0$

for the nodes at the stream boundary $\left(a b b^{\prime} c^{\prime} c d\right)$ we have $\psi_{i, j}=0 ; \zeta_{i, j}=0 ; u_{i, j}=0 ; v_{i, j}=0$

for the nodes at the stream boundaries $(A B)$ and $(c d)$ we have

$\zeta_{i, j}=2\left(\psi_{i, j-1}-\psi_{i, j}\right) / \eta^{2}$ for the nodes at the stream boundary $\left(B^{\prime} C^{\prime}\right)$ we have

$\zeta_{i, j}=2\left(\psi_{i+1, j}-\psi_{i, j}\right) / \xi^{2}$

for the nodes at the stream boundaries $(C D)$ and $(a b)$ we have

$\zeta_{i, j}=2\left(\psi_{i, j+1}-\psi_{i, j}\right) / \eta^{2}$

the following $\mathrm{BC}$ for dimensionless curl function $\zeta$, written for the nodes, belonging to die wall $\left(b^{\prime} c^{\prime}\right)$, must be satisfied

$\zeta_{i, j}=2\left(\psi_{i-1, j}-\psi_{i, j}\right) / \eta^{2}$

for the nodes at the stream boundary $\left(b b^{\prime}\right)$ we have

$\zeta_{i, j}=2\left(\psi_{i-1, j}+\psi_{i, j+1}-2 \psi_{i, j}\right) / \eta^{2}$ 

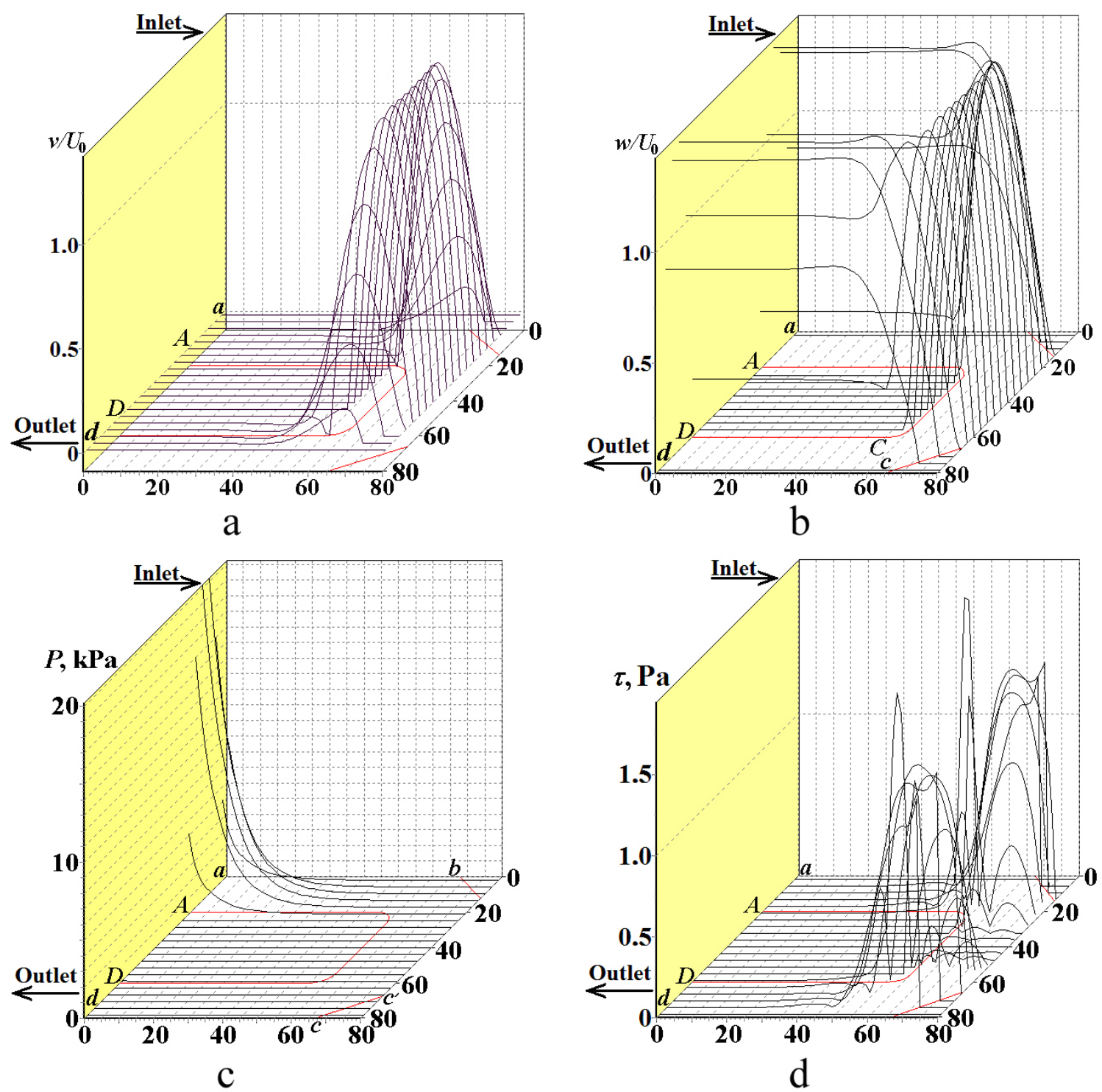

Fig. 4. CFD-derived dimensionless v-component of flow velocity w (a), dimensionless flow velocity w (b), dimensional punching pressure (c), and dimensional tangential stress (d), derived for ECMAE of plasticine viscous fluid through the U-shaped slanted ECMAE die, where the entrance $(\mathrm{aA})$ and the exit $(\mathrm{dD})$ are at the left.

for the nodes at the stream boundary $\left(c^{\prime} c\right)$ we have

$\zeta_{i, j}=2\left(\psi_{i-1, j}+\psi_{i, j-1}-2 \psi_{i, j}\right) / \eta^{2}$

for the nodes at the stream boundary $\left(B B^{\prime}\right)$ we have

$\zeta_{i, j}=2\left(\psi_{i+1, j}+\psi_{i, j-1}-2 \psi_{i, j}\right) / \eta^{2}$

for the nodes at the stream boundary $\left(C^{\prime} C\right)$ we have

$\zeta_{i, j}=2\left(\psi_{i-1, j}+\psi_{i, j-1}-2 \psi_{i, j}\right) / \eta^{2}$

for the angular points, located in the vertices of the “concave angles" $b, b$ ', $c, c$ ' we have

$\zeta_{i, j}=0$ for the angular point $B$ in the difference equation, written for the node $(i, j-1)$ we have

$\zeta_{i, j}=2\left(\psi_{i, j-1}-1\right) / \eta^{2}$

for the angular point $B$ in the difference equation, written for the node $(i+1, j)$ we have:

$\zeta_{i, j}=2\left(\psi_{i+1, j-1}-1\right) / \xi^{2}$

for the angular point $C$ in the difference equation, written for the node $(i, j+1)$ we have

$\zeta_{i, j}=2\left(\psi_{i, j+1}-1\right) / \eta^{2}$ 
for the angular point $C$ in the difference equation, written for the node $(i+1, j)$ we have

$\zeta_{i, j}=2\left(\psi_{i+1, j+1}-1\right) / \xi^{2}$

for the angular point $B$ ' in the difference equation, written for the node $(i+1, j)$ we have

$\zeta_{i, j}=2\left(\psi_{i+1, j}-1\right) / \xi^{2}$

for the angular point $B$ ' in the difference equation, written for the node $(i, j-1)$ we have

$\zeta_{i, j}=2\left(\psi_{i+1, j-1}-1\right) / \xi^{2}$

for the angular point $C^{\prime}$ in the difference equation, written for the node $(i+1, j)$ we have (20).

for the angular point $C^{\prime}$ in the difference equation, written for the node $(i, j+1)$ we have (19).

for the nodes at the entrance $(A a)$ of the multiple-angle die we have

$\psi_{i<20, j}=\psi_{20, j} ; u_{i<20, j}=u_{20, j} ; v_{i<20, j}=v_{20, j}$

For the nodes at the exit $(D d)$ of the multiple-angle die we have (22).

In the difference equations (5)-(22) parameters $\xi ; \eta$ are the steps of the finite-difference mesh. This article is focused on the CFD description of local flow of polymer workpiece through a modified U-shaped die during ECMAE, where the transient period of viscous flow is $43.241 \mathrm{~s}$ in Figs. $(3-4)$. The numerical computation results of the numerical integration of curl transfer equation (2)-(3) with initial (4) and boundary conditions (5)-(22) for the fields of the flow lines and energy-power parameters are outlined in Figs. $(3-4)$ for the following numerical values:

the dimensional width of inlet, outlet and transitional ECMAE die channel is $\bar{a}=40 \mathrm{~mm}$;

the dimensional density of the viscous plasticine physical model of extruded polymer material is $\bar{\rho}=1850 \mathrm{~kg} / \mathrm{m}^{3}$;

the dimensional plasticine yield strength is $\bar{\sigma}_{s}=217 \mathrm{kPa}$ (Sofuoglu et al., 2000) $\left[{ }^{12}\right]$;

the dimensional specific heat capacity of viscous plasticine material is $\bar{c}=1.004 \mathrm{~kJ} /(\mathrm{kg} \bullet \mathrm{K})$ (Chijiwa et al., 1981) $^{(2)}$;

the dimensional thermal conductivity is $\bar{\lambda}=0.7 \mathrm{~J} /$ $(\mathrm{m} \bullet \mathrm{s} \bullet \mathrm{K})\left(\right.$ Chijiwa et al., 1981) ${ }^{2}$;

the dimensional dynamic viscosity for viscous Newtonian fluid model of the plasticine workpiece is $\bar{\eta}_{v i s}=135 \mathrm{~Pa} \cdot \mathrm{s}$;

the dimensional kinematic viscosity for viscous Newtonian fluid model of the plasticine workpiece ECAE is $\bar{v}_{v i s}=\bar{\eta}_{v i s} / \bar{\rho}=135 / 1850=0.0729 \mathrm{~m}^{2} / \mathrm{s}$;

the dimensional characteristic average ECMAE punching velocity i.e. the workpiece velocity in the entrance channel $(A B a b)$ of the ECMAE die is $\bar{U}_{0}=0.27 \cdot 10^{-3} \mathrm{~m} / \mathrm{s}$; the dimensionless Reynolds number is

$\mathbf{R e}=\bar{U}_{0} \bar{a} \bar{\rho} / \bar{\eta}_{v i s}=\bar{U}_{0} \bar{a} / \bar{v}_{v i s}=\left(0.27 \cdot 10^{-3} \cdot 40 \cdot 10^{-3}\right) / 0.0729=1.48 \cdot 10^{-4} ;$

the dimensional time moment for the first isochrone building is $\overline{t_{l}}=100 \mathrm{~s}$;

the maximum value of dimensionless curl is $\zeta=15$;

the dimensional curl is

$\bar{\zeta}=\zeta \cdot \bar{U}_{0} / \bar{a}=\left(15 \cdot 0.27 \cdot 10^{-3} \mathrm{~m} / \mathrm{s}\right) /\left(40 \cdot 10^{-3} \mathrm{~m}\right)=101.25 \cdot 10^{-3} \mathrm{~s}^{-1}$ the dimensional average angular velocity of rotation for processed viscous material elementary volume is $\bar{\omega}=|\operatorname{rot} \underset{\mathbf{w}}{r}| / 2=\bar{\zeta} / 2=50.625 \cdot 10^{-3} s^{-1}$;

the half number of coordinate steps along the $x$-and $y$ - axes is $q=20$;

the coordinate increment is $2 \mathrm{~mm}$;

the dimensional time iteration step is $\bar{t}_{i t}=74 \mathrm{~ms}$;

the number of coordinate steps along the $x$ - and $y$-axes is $2 \bullet q=40$;

the relative error of iterations is $e=1 / 1000$.

The authors' written computer code for the numerical integration of the boundary value problem for curl transfer equation (2)-(3) with initial (4) and boundary conditions (5)-(22) has been proposed and practically realized by the authors with Object Pascal language.

\section{Discussion of Derived Numerical Simulation Results}

The CFD problem (2)-(22) implicitly takes into account the maximum value of contact friction between the viscous amorphous polymer workpiece material and the walls of the U-shaped slanted die $a b b^{\prime} c^{\prime} c d-A B B^{\prime} C^{\prime} C D$ by the definition of full sticking during viscous fluid flow. Note that there is an influence of instabilities of numerical solutions of the CFD problem (2)-(22) at the entrance $A a$ and at the exit $D d$ of the viscous flow on the accuracy of the numerical integration results in Figs. $(3-4)$.

Instabilities of numerical finite-difference solutions of the CFD problem (2)-(22), which appear at the inlet boundary (Aa) of the U-shaped slanted die $a b b^{\prime} c^{\prime} c d-A B B^{\prime} C^{\prime} C D$, propagate in the punching direction along $U_{0}$, i.e. move downstream. Instabilities of numerical solutions of the CFD problem (2)- (22), which appear at the outlet frontiers (Dd), move toward the material flow direction, i.e. propagate upstream. The numerical solutions (Figs. $(3-4))$ for the ECMAE through the U-shaped slanted die $a b b^{\prime} c^{\prime} c d-A B B^{\prime} C^{\prime} C D$ satisfactorily agree with the physical simulation results (Figs. (1 - 2)) only when the inlet boundary (Aa) and outlet boundary (Dd) of the viscous flows are located well away from the studied zone $\left(B B^{\prime} C^{\prime} C-b b^{\prime} c^{\prime} c\right)$ of the transient die channel, i.e. ideally the channel inlet (Aa) and outlet (Dd) have to be infinitely distant from the die channel transient zone $\left(B B^{\prime} C^{\prime} C-b b^{\prime} c^{\prime} c\right)$. So in order to protect the numerical solutions from numerical distortions, caused by instabilities at the inlet zone (Aa), the distribution 2D and 3D plots of energy-power ECMAE parameters in (Figs. $(3-4))$ have been derived assuming that the initial solution points are 
chosen remote from the coordinate grid inlet boundary (Aa) of the viscous flow $(i=0)$ and were chosen 20 cells away from the Aa boundary. Similarly for all 2D and 3D plots in Figs. $(3-4)$ the derived numerical fields have been truncated 20 cells away from the outlet (Dd) finite-difference boundary $(i=80)$ in order to remove the computational distortions caused by the outlet (Dd) instabilities. So the finite difference grid cells near the inlet and outlet have been rejected from the plots in Figs. $(3-4)$. We will now analyze and compare the results of the physical (Figs. $(1-2))$ and numerical (Figs. $(3-4))$ experiments.

The proposed die geometry in (Figs. $(1-4))$ has two slanted zones $b b^{\prime} B B^{\prime}$ and $c^{\prime} c C^{\prime} C$, which prevent dead zone formation in the vicinity of truncated external corners during viscous flow of the workpiece model through the U-shaped slanted die. The absence of the dead zones is shown in experimental photos (Figs. $(1(b, c) \& 2(a, c)))$, in computational flow lines (Fig. 3a), and in computational flow function $\psi$ diagrams (Fig. 3c). The experimentally-derived plots in Figs. (1(b, c) \& 2(a, c)) successively illustrate localization of zones with maximum values of logarithmic strain intensities.

The experimental 2D diagrams in Figs. (1(d) \& 2(b, d)) show the dynamics of displacement of the deformation zone within the deformed material as the workpiece is punched through the U-shaped slanted die during ECMAE. The first experimentally-derived strain diagram in Fig. 1d shows that at the initial stage of the flow the deformation zone is located in the vicinity of the line $b b$ ', i.e. includes the "external fibers" of deformed material, which are adjacent to transitional left lower slant $b b^{\prime}$. The second experimentally-derived strain diagram in Fig. $2 b$ indicates that at the final stage of filling the transition channel $b^{\prime} c^{\prime} B^{\prime} C^{\prime}$ ' of the $\mathrm{U}$ - shaped die the deformation zone within the workpiece volume completely moves to the vicinity of the horizontal internal transition zone $B$ ' $C$ ', i.e. the deformation zone occupies "internal fibers" of deformable material $B^{\prime} C^{\prime}$ ', and, so, the deformation zone goes beyond the $1^{\text {st }}$ slanted zone $b b^{\prime} B B^{\prime}$ '. The third experimentally-derived strain diagram in Fig. $2 d$ shows the initial stage of filling the outlet channel $c d C D$ of the $\mathrm{U}$ - shaped die and shows the displacement of the deformation zone in the vicinity of the horizontal external transition wall $b$ ' $c$ ', i.e. the new localization of the deformation zone includes the "external fibers" of deformed material in the vicinity of the flow border $b^{\prime} c$ '. The proposed original physical simulation, outlined in Figs. $(1-2)$, shows the dynamics of successive deformation working of the workpiece by punching and "mixing" both "external" and "internal" fibers of the long initial workpiece, that provides accumulation of the large shear strains for every pass of ECMAE through the U-shaped die.

The CFD-derived diagrams for flow velocities (Figs. (3(d) \& 4(a, b))) and tangential stresses (Fig. 4d) additionally show that as the workpiece goes to the outlet channel of the U-shaped die, the formation of velocity and tangential stress gradients occur within the workpiece volume and localization of these gradients exactly corresponds to the zones of the two slants $b b^{\prime} B B^{\prime}$ ' and $c^{\prime} c C^{\prime} C$. The observable formation of tangential stress gradients on the CFD-derived diagram in Fig. 4d is the hydrodynamic illustration of the appearance of the rotational deformation modes, which are visually observable in the experiment, shown in Figs. $(1-2)$.

The combined use of the physical simulation (Figs. (1 - 2)) and numerical CFD techniques (Figs. $(3-4))$ provides new experimental-theoretical results, which illustrate the dynamics of local material flow during SPD with ECMAE through truncated U-shaped dies. The proposed CFD-based approach (Figs. $(3-4))$ is appropriate for the analysis of ECMAE through the multiple-angled dies with more complex geometries of $\mathrm{S}-, \mathrm{T}-, \mathrm{X}-\mathrm{W}-$ types, taking into account the availability of the movable inlet, transitional, and outlet die walls.

\section{Conclusions}

The derived experimental-theoretical results allow the conclusion, that the geometric realization of the parallel slants in the two-turn truncated U-shaped die results in localization of the maximum tangential stresses within the workpiece worked material in the vicinity of the parallel slants during Equal Channel Multiple Angular Extrusion.

The derived experimental-theoretical results are grounded on the empirical analysis of the initial circular gridlines, and on the results of numerical finite-difference solution of the Navier-Stokes equations in curl transfer form for the local viscous flow of incompressible Newtonian viscous fluid through the truncated slanted multiple-angled U-shaped die.

The derived experimental-theoretical results enhance the understanding of local flow dynamics and the character of the formation of the rotational modes of deformations during Equal Channel Multiple Angle Extrusion through a truncated slanted multiple-angled U-shaped die. These results may lead to further applications to the analysis of shear flows in multiple-angled dies of more complex geometry with additional kinematical constraints in the shape of the moving walls.

\section{Disclosure}

The submission of the authors' paper implies that it has not been previously published, that it is not under consideration for publication elsewhere, and that it will not be published elsewhere in the same form without the written permission of the editors.

\section{Conflict of Interests}

The authors Alexander V. Perig and Nikolai N. Golodenko declare that there is no conflict of interests regarding the publication of this paper.

\section{Authors' contributions}

All authors participated in the design of this work and performed equally. All authors read and approved the final manuscript.

\section{Compliance with ethical guidelines}

Competing interests. The authors declare that they have no competing interests.

\section{Acknowledgements}

Authors thank "anonymous" referees for their valuable notes and suggestions. Authors are thankful to Materials Research (São Carlos) Editors and SciELO Team for this great opportunity to publish our original research at your respectful periodical Materials Research (São Carlos) under a Creative Commons License. 


\section{References}

1. Beloshenko VA, Voznyak A, Voznyak Y. Solid-phase extrusion of polyamide under simple shear. Polymer Science Series A. 2009;51(8):916-922. http://dx.doi.org/10.1134/S0965545X09080112

2. Chijiiwa K, Hatamura Y, Hasegawa N. Characteristics of plasticine used in the simulation of slab in rolling and continuous casting. Transactions of the Iron and Steel Institute of Japan. 1981;21(3):178-186. http://dx.doi.org/10.2355/ isijinternational1966.21.178

3. Minakowski P. Fluid model of crystal plasticity: numerical simulations of 2-turn equal channel angular extrusion. Technische Mechanik. 2014;34(3-4):213-221. http://www.uni-magdeburg. de/ifme/zeitschrift_tm/02_HTML_Inhalt/2014.htm

4. Oswald P. Rheophysics. The deformation and flow of matter. New York: Cambridge University Press; 2009.

5. Perig AV, Laptev AM, Golodenko NN, Erfort Yu A, Bondarenko EA. Equal channel angular extrusion of soft solids. Materials Science and Engineering A. 2010;527(16-17):3769-3776. http:// dx.doi.org/10.1016/j.msea.2010.03.043

6. Perig AV, Golodenko NN. CFD Simulation of ECAE through a multiple-angle die with a movable inlet wall. Chemical Engineering Communications. 2014;201(9):1221-1239. http:// dx.doi.org/10.1080/00986445.2014.894509

7. Perig AV, Golodenko NN. CFD 2D simulation of viscous flow during ECAE through a rectangular die with parallel slants.
International Journal of Advanced Manufacturing Technology. 2014;74(5-8):943-962. http://dx.doi.org/10.1007/s00170-014$5827-2$

8. Perig AV, Golodenko NN. ECAP process improvement based on the design of rational inclined punch shapes for the acuteangled Segal 20-dies: CFD 2-D description of dead zone reduction. Mechanical Science. 2015;6(1):41-49. http://dx.doi. org/10.5194/ms-6-41-2015

9. Perig AV. Two-parameter rigid block approach to upper bound analysis of equal channel angular extrusion through a Segal 20-Die. Materials Research. 2015;18(3):628-638. http://dx.doi. org/10.1590/1516-1439.004215

10. Rejaeian M, Aghaie-Khafri M. Study of ECAP based on stream function. Mechanical Science. 2014;76:27-34. http://dx.doi. org/10.1016/j.mechmat.2014.05.004

11. Roache PJ. Computational Fluid Dynamics. Albuquerque: Hermosa Publishers; 1976.

12. Sofuoglu H, Rasty J. Flow behavior of plasticine used in physical modeling of metal forming processes. Tribology International. 2000;33(8):523-529. http://dx.doi.org/10.1016/ S0301-679X(00)00092-X

13. Wang JP. A new evaluation to friction analysis for the ring test. International Journal of Machine Tools and Manufacture. 2001;41(3):311-324. http://dx.doi.org/10.1016/S08906955(00)00088-2. 